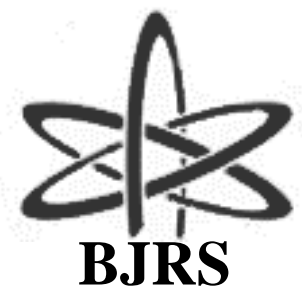

BRAZILIAN JOURNAL

$\mathrm{OF}$

RADIATION SCIENCES

08-01A (2020) 01-12

\title{
Uso de modelagem computacional para avaliação da exposição médica e ocupacional durante procedimento de cardiologia intervencionista
}

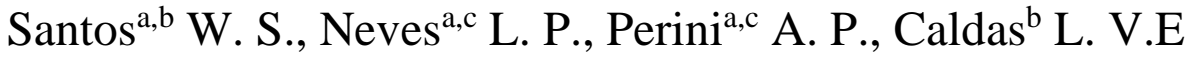 \\ ${ }^{a}$ Instituto de Física - Universidade Federal de Uberlândia (INFIS/UFU), Av. João Naves de Ávila, 2121, 38400-902 \\ Uberlândia, $M G$, Brasil. \\ ${ }^{b}$ Instituto de Pesquisas Energéticas e Nucleares (IPEN / CNEN - SP) Av. Professor Lineu Prestes, 2242, 05508-000 \\ São Paulo, SP, Brasil. \\ ${ }^{c}$ Programa de Pós-Graduação em Engenharia Biomédica, Faculdade de Engenharia Elétrica - Universidade Federal \\ de Uberlândia (PPGEB/UFU), Av. João Naves de Ávila 2121, 38400-902 Uberlândia, MG, Brasil. \\ william@ufu.br
}

\section{RESUMO}

A cardiologia intervencionista (CI) é uma modalidade comum que utiliza imagens fluoroscópicas para o diagnóstico e a terapia de problemas cardiovasculares. Embora os equipamentos de fluoroscopia mais modernos empreguem sistema de redução de dose, além de feixes bem colimados, a radiação espalhada atinge órgãos circundantes do paciente que não estão localizados no feixe primário de radiação. A radiação espalhada pelo corpo do paciente acarreta a exposição ocupacional do médico intervencionista. A avaliação das exposições ocupacional e médica é importante para a determinação dos riscos e das consequências biológicas em diferentes órgãos e tecidos. Neste trabalho, a avalição foi feita por meio do conceito de coeficiente de conversão (CC). Para quantificar os valores de CC, foi utilizado o programa computacional MCNPX e o simulador antropomórfico masculino virtual adulto (MASH3), para representar o paciente e o médico. Os resultados comprovaram a eficiência da proteção dos equipamentos suspensos. Quando não utilizados, um aumento dos valores de CC de dose efetiva podem ser superiores a $\mathbf{1 0 0 0 \%}$.

Palavras-chave: Cardiologia intervencionista, simulação de Monte Carlo, simulador antropomórfico virtual. 


\begin{abstract}
Interventional cardiology (IC) is a common modality that uses fluoroscopic images for the diagnosis and therapy of cardiovascular problems. While modern fluoroscopy equipments presents dose reduction systems, as well as collimated beams, the scattered radiation reaches surrounding patient organs that are not located in the primary radiation beam. Scattered radiation throughout the patient body will result in occupational exposure of the interventionist physician. The evaluation of occupational and medical exposure is important in assessing the risk and biological consequences in different organs and tissues. In this work, the evaluation was done through the concept of conversion coefficient (CC). To determine the $\mathrm{CC}$ values, the MCNPX computational program and the adult male virtual anthropomorphic phantom (MASH3) were used to represent the patient and the physician. The results proved the efficiency of the protection of suspended equipment. When not used, an increase in the $\mathrm{CC}$ values to effective dose may be larger than $1000 \%$.
\end{abstract}

Keywords: Interventional cardiology, Monte Carlo simulation, virtual anthropomorphic phantom .

\title{
1. INTRODUÇÃO
}

A cardiologia intervencionista (CI) é um ramo da medicina que utiliza imagens fluoroscópicas para documentar, acompanhar, diagnosticar e tratar pacientes com problemas cardíacos. A principal vantagem é a redução dos riscos de contaminação, complicação cirúrgica e de efeitos colaterais. A visualização em tempo real é possível graças à técnica de fluoroscopia. Nesse tipo de procedimento, uma boa qualidade da imagem é conseguida utilizando agentes de contraste intravenosos e um sistema de melhoramento de imagem. Embora sejam enormes os benefícios para o paciente, a CI é caracterizada por altas doses de radiação, tanto para o paciente quanto para o médico intervencionista. Dentro da área da medicina, os profissionais que realizam procedimentos de CI são os mais expostos à radiação [1,2]. Por essa razão, há muito interesse relacionado à pesquisa sobre este assunto para o desenvolvimento de métodos de estimativa de dose de pacientes e do médico intervencionista em CI [3,4,5,6]. Szumska et al. [5] avaliaram a exposição da equipe médica durante procedimentos de CI e não encontraram uma correlação significativa entre os valores de doses para o paciente e o médico intervencionista. Isso significa que as doses para o médico intervencionista não podem ser estimadas com base nas doses recebidas pelo paciente. Os valores 
medidos, atualmente, pelos detectores do aparelho de raios-X como kerma no ar num ponto de referência e produto dose-área (PDA), estimado num plano perpendicular do feixe de raios-X, podem servir apenas como parâmetros de avaliação da dose total recebida pelo paciente, mas para o médico intervencionista, correlações desse tipo não são possíveis, devido às alterações de um conjunto de parâmetros radiográficos, como espessura da região examinada, angulação do feixe, variação de tensão do tubo e da filtração, que são fatores que modificam a radiação espalhada.

Em CI a principal fonte de exposição do médico intervencionista é a radiação espalhada pelo corpo do paciente. Para minimizar a exposição do médico intervencionista, é recomendada a utilização de equipamentos de proteção individual como aventais plumbíferos, óculos plumbíferos e protetores de tireoide, além de proteção adicional, como as proteções móveis suspensas. A determinação experimental do efeito da radiação espalhada no valor da dose ocupacional pode ser feita usando simuladores antropomórficos físicos. Esta solução, entretanto, é mais complexa e dispendiosa, sendo o Método de Monte Carlo uma solução mais acessível. Neste caso, são empregados simuladores antropomórficos virtuais. Neste sentido, o principal objetivo deste trabalho foi utilizar simulações Monte Carlo para avaliar a exposição ocupacional em termos de coeficiente de conversão de dose efetiva $(\mathrm{CC}[\mathrm{E}])$ e dose equivalente $\left(\mathrm{CC}\left[\mathrm{H}_{\mathrm{T}}\right]\right)$ para os órgãos e tecidos do paciente e do médico intervencionista, nomalizados pelo produto dose-área (PDA).

\section{MATERIAIS E MÉTODOS}

Neste estudo, o médico intervencionista e o paciente foram representados pelo simulador antropomórfico virtual MASH3, que foi construído com base nas características antropométricas do homem de referência definidos pela ICRP 89, que possui $176 \mathrm{~cm}$ de altura e $73 \mathrm{~kg}$ de massa córporea [7,8]. Além da modelagem dos principais equipamentos da sala de CI, como cama do paciente, cortina de chumbo, mesa de instrumentação cirúrgica, tubo de raios-X, detector flat-panel, foram modelados equipamentos de proteção individual e coletiva, como óculos plumbíferos, protetores de tireóide e barreiras suspensas com $0,5 \mathrm{mmPb}$ de espessura cada. Além do médico e do paciente, a Figura 1 mostra a maioria dos equipamentos típicos de uma sala de CI e também a vista das projeções do feixe empregadas neste trabalho. A Figura 2 mostra os principais equipamentos de proteção individual utilizados pelo o simulador antropomórfico virtual MASH3 como, por exemplo, 
avental plumbífero, óculos plumbíferos e protetor de tireoide. A maca foi confeccionada com fibra de carbono e possui largura de $70 \mathrm{~cm}$, espessura de $5 \mathrm{~cm}$ e comprimento de $200 \mathrm{~cm}$.

O código de transporte de radiação MCNPX 2.7.0 (Monte-Carlo N-Particle eXtended) [9] foi empregado nas simulações. Foi utilizada uma fonte pontual de raios-X emitindo fótons em quatro projeções de feixe: pôstero-anterior (PA), lateral esquerdo (LAO90), oblíquo lateral esquerdo e direito (LAO65 e RAO65). As distâncias foco-superfície (DFS) para estas projeções foram 60, 50, 56 e $53 \mathrm{~cm}$, respectivamente. A tensão no tubo fio de $80 \mathrm{kVp}$, filtração total de $3,5 \mathrm{mmAl}$ e ângulo anódico de $12^{\circ}$. Os espectros de fótons foram gerados com o software SRS 78 [10]. Para manter as incertezas aceitáveis, em todos os cenários de irradiação foram utilizadas um bilhão de histórias de partículas.
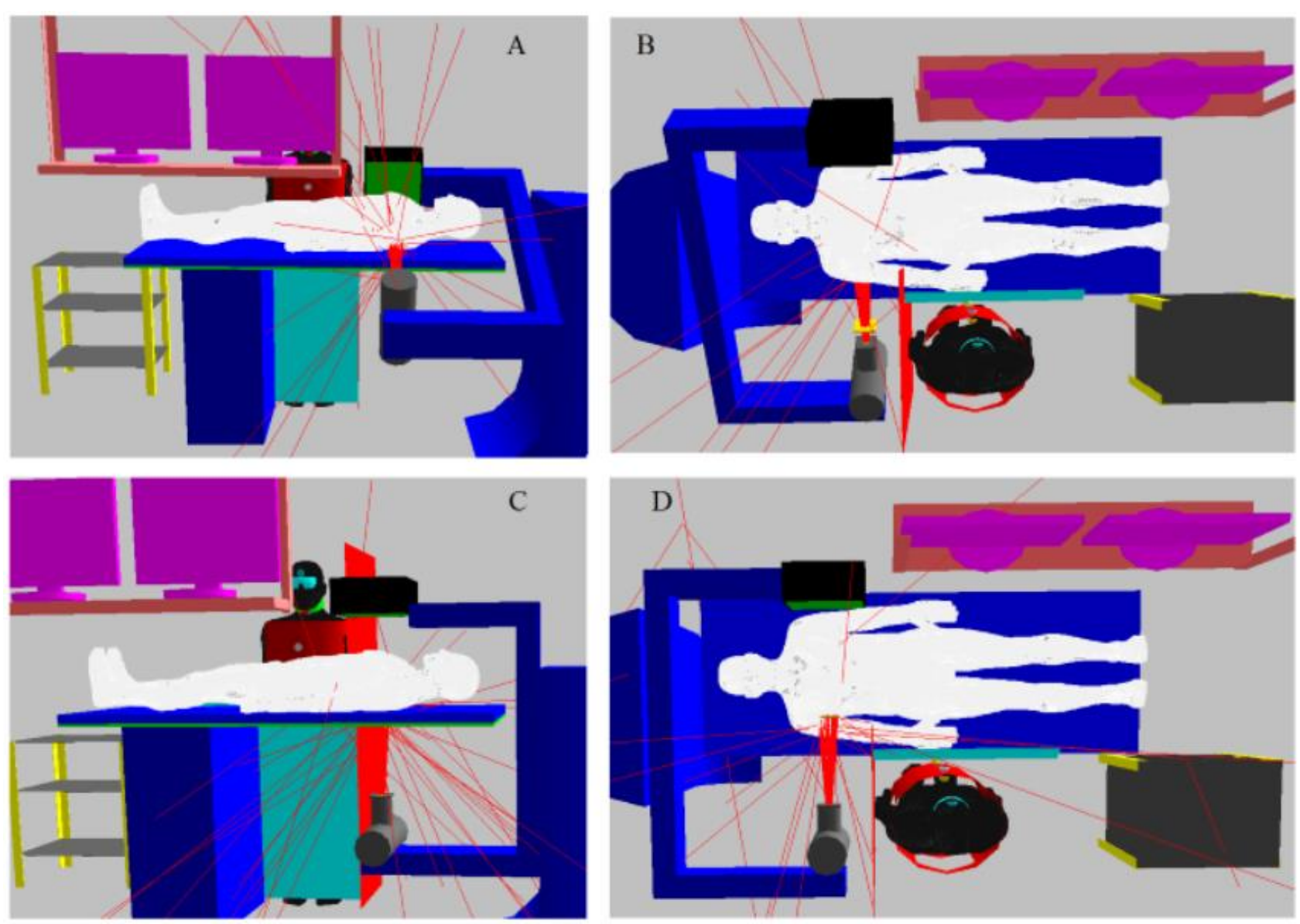

Figura 1. Vista das direções de feixe oblíqua anterior (A) direita (RAO65) e (B) esquerda (LAO65); (C) pôstero-anterior (PA) e (D) lateral esquerda (LAO90). 


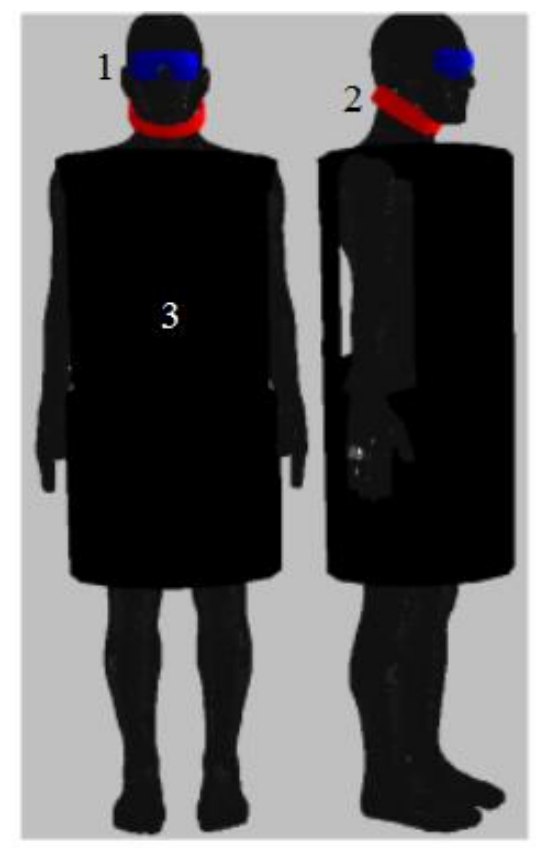

Figura 2. Equipamentos de proteção individual modelados: (1) Óculos plunbíferos, (2) protetor de tireoide e (3) avental plumbífero.

Durante os procedimentos de CI é importante relatar os valores de PDA, pois a avaliação da exposição do paciente será interpretada com base nos valores dessa grandeza. Por conseguinte, todos os equipamentos de raios-X vêm de fábrica equipados com uma câmara de ionização de transmissão, instalada na saída do tubo, para estimar o PDA e, por isso, neste estudo também foi modelado um objeto preenchido com ar atmosférico e posicionado na saída do tubo para simular a câmara de ionização.

A determinação dos valores de CC[E] foi feita com base nas recomendações da International Commission on Radiological Protection [ICRP 103] para dose efetiva. Essa é definida como a soma ponderada das doses equivalentes de 14 órgãos e tecidos críticos do corpo: medula vermelha, cólon, pulmão, estômago, mamas, gônadas, bexiga, esôfago, fígado, tireoide, superfície óssea, cérebro, glândulas salivares, pele e demais órgãos (glândulas adrenais, região extratorácica, vesícula biliar, coração, rins, nódulos linfáticos, músculo, mucosa oral, pâncreas, próstata, intestino delgado, baço, timo e útero) [11].

Os valores de $\mathrm{CC}\left[\mathrm{H}_{\mathrm{T}}\right]$ foram determinados conforme mostra a Equação (1), e os valores de $\mathrm{CC}[\mathrm{E}]$, conforme mostra a Equação (2): 


$$
\begin{gathered}
C C\left[H_{T}\right]=\frac{H_{T} * w_{T}}{P D A} \\
C C[E]=\sum_{T} C C\left[H_{T}\right]
\end{gathered}
$$

onde $w_{T}$ é o fator de ponderação para o órgão/tecido $\mathrm{T}$ e $\mathrm{H}_{\mathrm{T}}$ é a dose equivalente. Como o valor recomendado do fator de ponderação da radiação para fóton é 1 para qualquer energia, a dose equivalente é a mesma que a dose absorvida.

Neste estudo, a energia máxima de fótons em procedimento de CI foi de $80 \mathrm{keV}$ e, por isso, os processos de interação mais prováveis e considerados foram a absorção fotoelétrica, com a possibilidade de emissão fluorescente e elétrons Auger, espalhamento coerente (Rayleigh) e incoerente (Compton). O transporte de fótons e elétrons foi realizado utilizando a biblioteca de seção de choque ENDF/B-VI8 do código MCNPX [9].

\section{RESULTADOS E DISCUSSÃO}

\subsection{Valores de $\mathrm{CC}[\mathrm{E}]$ e $\mathrm{CC}\left[\mathrm{H}_{\mathrm{T}}\right]$}

Os resultados dos $\mathrm{CC}[\mathrm{E}]$ e $\mathrm{CC}\left[\mathrm{H}_{\mathrm{T}}\right]$ para o médico e o paciente estão apresentados nas Tabelas 1 e 2, respectivamente. É possível observar que o médico intervencionista está mais exposto à radiação quando a projeção LAO90 (Figura 1 D) é utilizada. Nas projeções LAO90 e LAO65 (Figura 1 B), o tubo de raios-X está localizado do lado do médico. A radiação retroespalhada do detector flat-panel e, principalmente, do corpo do paciente, representa a principal contribuição para o aumento da exposição do médico intervencionista. Comparada com a projeção PA (Figura $1 \mathrm{C}$ ) e RAO65 (Figura 1 A), que obtiveram os menores valores de CC[E], a projeção LAO90 teve um aumento de 3 vezes, enquanto a lente dos olhos apresentou um aumento nos valores de $\mathrm{CC}\left[\mathrm{H}_{\mathrm{T}}\right]$ de 2 e 2,7 vezes, respectivamente. Para o paciente, a projeção mais crítica foi a LAO90. Este resultado era esperado, pois o feixe é projetado diretamente sobre o paciente. Nas outras situações, o tubo de raios-X está embaixo da mesa do paciente, que atenua parte dos fótons que chegariam aos órgãos do paciente. Neste sentido, é importante fazer um comentário: neste estudo foi utilizada uma única tensão do tubo de $80 \mathrm{kVp}$. Na prática clínica, a maioria dos equipamentos de raios-X ajusta automaticamente a exposição à radiação por meio de um sistema denominado de controle 
automático de exposição (CAE), que não foi considerado neste estudo. O CAE é capaz de detectar as variações de sinal produzido no receptor de imagem, e ajusta o gerador de raios-X para aumentar ou diminuir os parâmetros radiográficos $(\mathrm{kV}, \mathrm{mA}$ e tempo de pulso), para que a imagem seja de qualidade consistente. Quando uma parte mais espessa do corpo do paciente está sendo examinada, ou quando o tubo de raios-X está embaixo da mesa, o equipamento de raios-X aumentará automaticamente os parâmetros radiográficos para manter a qualidade da imagem.

Dependendo da complexidade do procedimento de CI, o tempo de fluoroscopia pode ser prolongado. Além disso, a concentração do feixe numa única projeção gera o risco de maior dose na pele do paciente, que ocasionalmente, pode exceder o limiar de $2 \mathrm{~Gy}$, acima do qual, podem surgir eritemas na pele e, por isso, recomenda-se monitorar as doses na entrada da pele do paciente (DEP). Os valores de $\mathrm{CC}\left[\mathrm{H}_{\mathrm{T}}\right]$ e $\mathrm{CC}[\mathrm{E}]$ para o médico intervencionista são apresentados na Tabela 1 e os valores para o paciente, na Tabela 2. Os valores de CC para dose de entrada na pele (CC[DEP]), também, são apresentados na Tabela 2.

Tabela 1. Coeficientes de conversão $\mathrm{CC}\left[\mathrm{H}_{\mathrm{T}}\right]$ e $\mathrm{CC}[\mathrm{E}]$ para o médico intervencionista. A incerteza Tipo A da simulação é apresentada entre parênteses (em \%).

\begin{tabular}{ccccc}
\hline Médico & \multicolumn{4}{c}{ CC[HT] $\left(\boldsymbol{\mu S v} / \mathbf{G y . c m} \mathbf{2}^{\mathbf{2}}\right.$} \\
\cline { 2 - 5 } Órgãos / tecidos & PA & LAO65 & RAO65 & LAO90 \\
\hline Medula vermelha & $4,1 \mathrm{E}-02(0,5)$ & $7,0 \mathrm{E}-02(0,4)$ & $3,7 \mathrm{E}-02(0,5)$ & $1,1 \mathrm{E}-01(0,3)$ \\
Cólon & $3,1 \mathrm{E}-03(5,1)$ & $1,8 \mathrm{E}-02(2,0)$ & $3,1 \mathrm{E}-03(5,3)$ & $3,0 \mathrm{E}-02(1,7)$ \\
Pulmão & $1,4 \mathrm{E}-02(1,7)$ & $2,6 \mathrm{E}-02(1,5)$ & $1,5 \mathrm{E}-02(2,0)$ & $5,7 \mathrm{E}-02(0,8)$ \\
Estômago & $7,1 \mathrm{E}-03(5,3)$ & $2,4 \mathrm{E}-02(3,0)$ & $4,7 \mathrm{E}-03(6,5)$ & $5,5 \mathrm{E}-02(2,0)$ \\
Mamas & $2,5 \mathrm{E}-02(5,5)$ & $1,8 \mathrm{E}-02(8,0)$ & $1,1 \mathrm{E}-02(10)$ & $4,1 \mathrm{E}-02(5,0)$ \\
Demais tecidos & $1,3 \mathrm{E}-03(0,5)$ & $2,4 \mathrm{E}-03(0,3)$ & $1,0 \mathrm{E}-03(0,5)$ & $3,9 \mathrm{E}-03(0,2)$ \\
Gônadas & $5,7 \mathrm{E}-03(16)$ & $4,6 \mathrm{E}-03(16)$ & $4,2 \mathrm{E}-03(16)$ & $3,6 \mathrm{E}-03(19)$ \\
Bexiga & $7,9 \mathrm{E}-04(24)$ & $2,9 \mathrm{E}-03(13)$ & $1,4 \mathrm{E}-03(17)$ & $3,4 \mathrm{E}-03(11)$ \\
Esôfago & $9,7 \mathrm{E}-03(8,0)$ & $1,6 \mathrm{E}-02(7,0)$ & $1,3 \mathrm{E}-02(7,0)$ & $3,4 \mathrm{E}-02(4,0)$ \\
Fígado & $4,6 \mathrm{E}-03(3,5)$ & $8,6 \mathrm{E}-03(3,0)$ & $3,0 \mathrm{E}-03(5,0)$ & $2,0 \mathrm{E}-02(2,0)$ \\
Tireoide & $2,3 \mathrm{E}-02(8,0)$ & $2,8 \mathrm{E}-02(7,5)$ & $5,4 \mathrm{E}-02(5,5)$ & $7,4 \mathrm{E}-02(4,5)$ \\
Superfície óssea & $1,5 \mathrm{E}-02(0,5)$ & $2,7 \mathrm{E}-02(0,5)$ & $1,4 \mathrm{E}-02(0,5)$ & $4,3 \mathrm{E}-02(0,3)$ \\
Cérebro & $4,1 \mathrm{E}-02(1,5)$ & $6,0 \mathrm{E}-02(1,2)$ & $4,1 \mathrm{E}-02(1,5)$ & $1,1 \mathrm{E}-01(1,0)$ \\
Glândulas salivares & $2,6 \mathrm{E}-02(2,0)$ & $3,5 \mathrm{E}-02(2,0)$ & $3,1 \mathrm{E}-02(2,0)$ & $6,4 \mathrm{E}-02(1,3)$ \\
Pele & $4,0 \mathrm{E}-02(0,5)$ & $6,6 \mathrm{E}-02(0,3)$ & $3,2 \mathrm{E}-02(0,4)$ & $9,7 \mathrm{E}-02(0,2)$ \\
Lentes dos olhos & $2,2 \mathrm{E}-01(6,5)$ & $2,6 \mathrm{E}-01(6,0)$ & $1,6 \mathrm{E}-01(7,3)$ & $4,2 \mathrm{E}-01(4,5)$ \\
Olhos & $1,8 \mathrm{E}-01(3,0)$ & $2,2 \mathrm{E}-01(3,0)$ & $1,4 \mathrm{E}-01(3,4)$ & $3,4 \mathrm{E}-01(2,0)$ \\
\hline CC[E] $\left(\mu\right.$ Sv/Gy.cm $\left.{ }^{2}\right)$ & $1,4 \mathrm{E}-02(2,0)$ & $2,3 \mathrm{E}-02(2,0)$ & $1,3 \mathrm{E}-02(2,0)$ & $4,5 \mathrm{E}-02(2,0)$ \\
\hline
\end{tabular}


Tabela 2. Coeficientes de conversão $\mathrm{CC}\left[\mathrm{H}_{\mathrm{T}}\right]$ e $\mathrm{CC}[\mathrm{E}]$ para o paciente. A incerteza Tipo A da simulação é apresentada entre parênteses (em \%).

\begin{tabular}{ccccc}
\hline Paciente & \multicolumn{4}{c}{ CC[HT] $\left.(\mathbf{m S v} / \mathbf{G y . c m})^{2}\right)$} \\
\cline { 2 - 5 } Órgãos / tecidos & PA & LAO65 & RAO65 & LAO90 \\
\hline Medula vermelha & $1,2 \mathrm{E}-01(0,1)$ & $1,1 \mathrm{E}-01(0,1)$ & $1,3 \mathrm{E}-01(0,1)$ & $1,2 \mathrm{E}-01(0,1)$ \\
Cólon & $3,3 \mathrm{E}-03(0,2)$ & $2,3 \mathrm{E}-03(0,2)$ & $4,0 \mathrm{E}-03(0,2)$ & $3,4 \mathrm{E}-03(0,2)$ \\
Pulmão & $4,0 \mathrm{E}-01(0,1)$ & $2,6 \mathrm{E}-01(0,1)$ & $2,6 \mathrm{E}-01(0,1)$ & $6,6 \mathrm{E}-01(0,1)$ \\
Estômago & $3,6 \mathrm{E}-02(0,1)$ & $1,9 \mathrm{E}-02(0,1)$ & $4,1 \mathrm{E}-02(0,1)$ & $4,1 \mathrm{E}-02(0,1)$ \\
Mamas & $7,1 \mathrm{E}-02(0,1)$ & $2,1 \mathrm{E}-02(0,2)$ & $2,1 \mathrm{E}-02(0,2)$ & $5,4 \mathrm{E}-02(0,2)$ \\
Demais tecidos & $8,6 \mathrm{E}-03(0,1)$ & $9,7 \mathrm{E}-03(0,1)$ & $1,1 \mathrm{E}-02(0,1)$ & $9,1 \mathrm{E}-03(0,1)$ \\
Gônadas & $2,4 \mathrm{E}-04(2,4)$ & $3,5 \mathrm{E}-04(2,0)$ & $3,5 \mathrm{E}-04(2,0)$ & $2,6 \mathrm{E}-04(2,0)$ \\
Bexiga & $5,8 \mathrm{E}-04(1,0)$ & $5,1 \mathrm{E}-04(1,0)$ & $4,8 \mathrm{E}-04(1,0)$ & $3,2 \mathrm{E}-04(1,2)$ \\
Esôfago & $4,1 \mathrm{E}-01(0,1)$ & $3,1 \mathrm{E}-01(0,1)$ & $2,9 \mathrm{E}-01(0,1)$ & $5,1 \mathrm{E}-01(0,1)$ \\
Fígado & $6,6 \mathrm{E}-02(0,1)$ & $5,1 \mathrm{E}-02(0,1)$ & $2,8 \mathrm{E}-02(0,1)$ & $1,2 \mathrm{E}-01(0,1)$ \\
Tireoide & $4,2 \mathrm{E}-02(0,2)$ & $3,3 \mathrm{E}-02(0,2)$ & $3,2 \mathrm{E}-02(0,2)$ & $4,9 \mathrm{E}-02(0,2)$ \\
Superfície óssea & $6,7 \mathrm{E}-02(0,1)$ & $4,3 \mathrm{E}-02(0,1)$ & $4,9 \mathrm{E}-02(0,1)$ & $5,0 \mathrm{E}-02(0,1)$ \\
Cérebro & $1,6 \mathrm{E}-03(0,2)$ & $1,1 \mathrm{E}-03(0,3)$ & $1,1 \mathrm{E}-03(0,3)$ & $7,2 \mathrm{E}-04(0,2)$ \\
Glândulas salivares & $2,1 \mathrm{E}-03(0,1)$ & $1,7 \mathrm{E}-03(0,3)$ & $1,7 \mathrm{E}-03(0,1)$ & $2,3 \mathrm{E}-03(0,1)$ \\
Pele & $4,0 \mathrm{E}-02(0,1)$ & $1,6 \mathrm{E}-01(0,1)$ & $1,3 \mathrm{E}-01(0,1)$ & $1,3 \mathrm{E}-01(0,3)$ \\
Lente dos olhos & $8,5 \mathrm{E}-04(3,7)$ & $6,3 \mathrm{E}-04(4,0)$ & $8,0 \mathrm{E}-04(4,0)$ & $1,1 \mathrm{E}-03(0,2)$ \\
Olhos & $7,2 \mathrm{E}-04(1,5)$ & $6,8 \mathrm{E}-04(1,5)$ & $7,7 \mathrm{E}-04(1,5)$ & $1,1 \mathrm{E}-03(0,1)$ \\
CC[DEP] & $8,1(0.1)$ & $8,7(0.1)$ & $8,0(0.1)$ & $6,8(0.1)$ \\
\hline CC[E] (mSv/Gy.cm $\left.{ }^{2}\right)$ & $1,0 \mathrm{E}-01(0,2)$ & $6,8 \mathrm{E}-02(0,2)$ & $7,2 \mathrm{E}-02(0,2)$ & $1,4 \mathrm{E}-01(0,2)$ \\
\hline
\end{tabular}

$\mathrm{Na}$ prática clínica, o monitoramento da dose em tempo real é algo difícil, uma vez que é impossível prever o local da exposição máxima antes do procedimento. $\mathrm{Na}$ ausência de medições, o valor de CC[DEP] apresentado na Tabela 2 pode ser usado para se estabelecer uma estimativa da dose de entrada na pele a partir dos valores de PDA, fornecidos no console do equipamento de raios-X. A média obtida neste estudo foi de $8,0 \mathrm{mGy} / \mathrm{Gy} . \mathrm{cm}^{2}$, que é consistente com resultados experimentais publicados na literatura, que estão no intervalo de 4 - 8 mGy/Gy.cm² $[12,13,14]$.

Mesmo não havendo um limite de dose efetiva para exposição médica, neste estudo optou-se por apresentar os valores de $\mathrm{CC}[\mathrm{E}]$, pois é importante avaliar o risco potencial de efeitos estocásticos, como câncer e leucemia [11]. O CC[E] médio de todas as projeções foi de $0,10 \mathrm{mGy} / \mathrm{Gy} \cdot \mathrm{cm}^{2}$, que é inferior aos resultados experimentais da literatura, $0,183 \mathrm{mSv} / \mathrm{Gy} \cdot \mathrm{cm}^{2}$ [15], $0,185 \mathrm{mSv} / \mathrm{Gy} \cdot \mathrm{cm}^{2}$ [16] e $0,137 \mathrm{mSv} / \mathrm{Gy} \cdot \mathrm{cm}^{2}$ [17]. Estas diferenças são justificadas diante da complexidade dos procedimentos de CI, como variação do ângulo de incidência do feixe, tamanho 
do campo, índice de massa corporal (IMC) do paciente e alterações da tensão no tubo. Esses são alguns dos fatores que alteram os valores de dose em órgãos e, consequentemente, a dose efetiva do paciente.

\subsection{Influência dos protetores suspensos nos valores de $\mathrm{CC}[\mathrm{E}]$ e de $\mathrm{CC}\left[\mathrm{H}_{\mathrm{T}}\right]_{\text {lente dos olhos }}$ do médico intervencionista}

Na Tabela 1 foram apresentados valores de CC para uma situação em que o médico intervencionista faz uso de barreira de proteção feita com vidro plumbífero. Nesta seção, é feita uma avaliação dosimétrica em que o médico não faz uso de protetores suspensos de vidros plumbíferos. Os valores dos $\mathrm{CC}[\mathrm{E}]$ e $\mathrm{CC}\left[\mathrm{H}_{\mathrm{T}}\right]_{\text {lente dos olhos, nesse tipo de configuração, estão }}$ apresentados na Tabela 3.

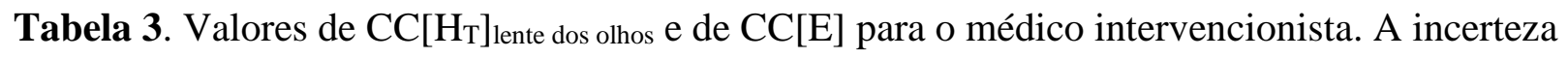
Tipo A da simulação é apresentada entre parênteses (em \%).

\begin{tabular}{ccccc}
\hline & \multicolumn{4}{c}{$\left(\boldsymbol{\mu S v} / \mathbf{G y . c m}{ }^{2}\right)$} \\
\cline { 2 - 5 } Médico & PA & LAO65 & RAO65 & LAO90 \\
\hline $\mathrm{CC}\left[\mathrm{H}_{\mathrm{T}}\right]_{\text {lente dos olhos }}$ & $7,0 \mathrm{E}-01(3,5)$ & $2,8(2,0)$ & $2,9 \mathrm{E}-01(6,0)$ & $6,5(1,0)$ \\
$\mathrm{CC}[\mathrm{E}]$ & $7,9 \mathrm{E}-02(1,0)$ & $3,3 \mathrm{E}-01(1,0)$ & $3,5 \mathrm{E}-02(1,0)$ & $5,5 \mathrm{E}-01(1,0)$ \\
\hline
\end{tabular}

Comparando os resultados dos valores de CC da Tabela 3 com os da Tabela 1, para todas as configurações avaliadas, nota-se que a não utilização de proteção suspensa resulta em aumento significativo da exposição ocupacional. Os resultados dos valores de $\mathrm{CC}\left[\mathrm{H}_{\mathrm{T}}\right]_{\text {lente dos olhos tiveram um }}$ aumento de 218\% (PA), 977\% (LAO65), 81\% (RAO65) e 1448\% (LAO90), quando não foram utilizadas proteções suspensas. Panorma semelhante é observado para os valores de CC[E], cujos aumentos foram de 464\% (PA), 1335\% (LAO65), 169\% (RAO65) e 1122\% (LAO90).

Um ponto importante deve ser destacado. Na prática clínica, o médico intervencionista pode utilizar protetores suspensos de diferentes modelos, formas, tamanhos e posições diferentes e, por isso, sua eficiência na redução da exposição pode variar significativamente em relação aos resultados apresentados neste estudo. Em todas as configurações avaliadas por este estudo, a forma, tamanho e posições dos protetores suspensos foram mantidas constantes. 


\section{CONCLUSÕES}

Neste trabalho, foi utilizado o simulador antropomórfico MASH3 e o código computacional MCNPX (2.7.0) para calcular os valores de $\mathrm{CC}[\mathrm{E}]$ e $\mathrm{CC}\left[\mathrm{H}_{\mathrm{T}}\right]$ para o médico intervencionista e paciente, num cenário típico de cardiologia intervencionista. Os resultados confirmam que a radiação espalhada pelo corpo do paciente é mais intensa na entrada do feixe de raios-X, isto é, no lado onde o tubo de raios-X está localizado (LAO65/LAO90). Além da projeção, outro fator importante na redução da exposição é a utilização dos protetores suspensos. Quando não são

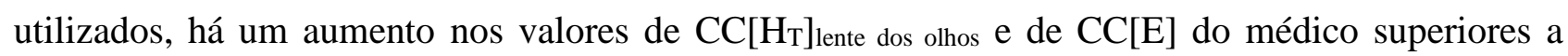
$1000 \%$ e, por isso, sempre que for possível, protetores suspensos devem ser usados. Neste sentido, é importante que os profissionais médicos envolvidos nos procedimentos de CI sejam orientados a usar os equipamentos de proteção suspensos corretamente.

\section{AGRADECIMENTOS}

Os autores gostariam de agradecer ao Dr. Richard Kramer por gentilmente fornecer o simulador antropomórfico virtual MASH3 usado neste trabalho. Este trabalho foi parcialmente financiado pelas agências brasileiras: Fundação de Amparo e Pesquisa do Estado de Minas Gerais (FAPEMIG, projetos $\mathrm{n}^{\circ}$ APQ-03049-15 e APQ-02934-15) e Conselho Nacional de Desenvolvimento Científico e Tecnológico (CNPq, projetos no 421603/2016-0, 420699/2016-3, 153177/2018-7 e 301335/2016$8)$.

\section{REFERENCIAS}

[1] BOZKURT, A.; BOR, D. Simultaneous determination of equivalent dose to organs and tissues of the patient and of the physician in interventional radiology using the Monte Carlo method. Phys Med Biol, v. 52, p. 317 - 330, 2007.

[2] FERRARI, P.; BECKER, F.; CARINOU, E.; CHUMAK, V.; FARAH, J.; JOVANOVIC, Z.; KRSTIC, D.; MORGUN, A.; PRINCIPI, S.; TELES, P. Monte Carlo study of the scattered radiation field near the eyes of the operator in interventional procedures. J Radiol Prot, v. 36, p. 902-921, 2016. 
[3] KOUKORAVA, C.; CARINOU, E.; FERRARI, P.; KRIM.; STRUELENS, L. Study of the parameters affecting operator doses in interventional radiology using Monte Carlo simulations. Radiat Meas, v. 46, p. 1216-1222, 2011.

[4] SANTOS, W. S.; BElinAtO, B.; PERINI, A.P.; CALDAS, L.V.E.; GAlEANO, D.C.; SANTOS, C.J.; NEVES, L. P. Occupational exposures during abdominal fluoroscopically guided interventional procedures for different patient sizes - A Monte Carlo approach. Physica Medica, v. 45 , p. 35-43, 2018.

[5] SZUMSKA, A.; KOPEC, R.; BUDZANOWSKI, M. Occupational doses of medical staff and their relation to patient exposure incurred in coronary angiography and intervention. Radiat Meas, v. 84 , p. $34-40,2016$.

[6] VANO, E.; KLEIMAN, N. J.; DURAN, A.; REHANI, M.; ECHEVERRI, D.; CABRERA, M. Risk for radiation cataract in interventional cardiology personnel. Radiat Res, v. 174, p. 490-495, 2010.

[7] CASSOLA, V.F.; DE LIMA, V.J.; KRAMER, R.; KHOURY, H. J. FASH and MASH: Female and male adult human phantoms based on polygon meSH surfaces. Part II. Dosimetric calculations. Phys Med Biol, v. 55, p. 163-189, 2010.

[8] ICRP 89. INTERNATIONAL COMMISSION ON RADIOLOGICAL PROTECTION. Basic anatomical and physics data for use in radiological protection: reference values. Pergamon Press. Oxford, 2003.

[9] PELOWITZ, D. B. MCNPX User`s Manual, version 2.7.0. Report LA-CP-11-00438. Los Alamos National Laboratory, 2011.

[10] CRANLEY, K.; GILMORE, B.J.; FOGARTY, G.W.A.; DESPONDS, L. Catalogue of diagnostic $x$-ray spectra and other data, Institute of Physics and Engineering in Medicine Report 78. (York: IPEM), 1997.

[11] ICRP 103. INTERNATIONAL COMMISSION ON RADIOLOGICAL PROTECTION. Recommendations of the ICRP publication 103. Elsevier, v. 37, p. 61-62, 2007.

[12] CHIDA, K.; SAITO, H.; OTANI, H.; KOHZUKI, M.; TAKAHASI, S.; YAMANDA, S.; SHIRATO, K.; ZUGUCHI, M. Relationship between fluoroscopic time, dose-area product, body weight, and maximum radiation skin dose in cardiac interventional procedures. AJR Am J Roentgenol, v. 186, p. 774-778, 2006. 
[13] QUAI, E.; PADOVANI, R.; PETERZOL, A.; VAÑO, E.; GUIBELALDE, E.; TOIVONEN, M. Maximum skin dose assessment in interventional cardiology: results in three different European hospitals. Eur Radiol, v. 13, p. 542 - 553, 2003.

[14] ALTHEN, J. N.; SANDBORG, M. Verification of indicated skin entrance air kerma for cardiac X-ray-guided intervention using gafchromic film. Radiat Prot Dosim, v. 15, p. 1 - 4, 2015. [15] BETSOU, S.; EFSTATHOPOULOS, E.P.; KATRITSIS, D.; FAULKNER, K.; PANAYIOTAKIS, G. Patient radiation doses during cardiac catheterization procedures. Br J Radiol, v. 71, p. 634-639, 1998.

[16] BOGAERT, E.; BACHER, K.; THIERENS, H. A. Large-scale multicentre study in Belgium of dose area product values and effective doses in interventional cardiology using contemporary x-ray equipment. Radiat Prot Dosim, v. 128, p. 312-323, 2007.

[17] STISOVA, V. Effective dose to patient during cardiac interventional procedures (Prague workplaces). Radiat Prot Dosim, v 111, p. 271-274, 2004. 